\title{
Rentabilidad del uso de agua marina desalinizada en la produc- ción de tomate de invernadero en Almería
}

\author{
Martínez-Granados, D. ${ }^{1}$, Marín-Membrive, P. ${ }^{2}$, Calatrava, J. ${ }^{3}$ \\ Investigador, E.T.S. de Ingeniería Agronómica, Universidad Politécnica de Cartagena, Cartagena; david.martinez@upct.es \\ Investigadora, Departamento de Ingeniería, Universidad de Almería, La Cañada de San Urbano, Almería; patriciamarin@ual.es \\ Catedrático de Universidad, E.T.S. de Ingeniería Agronómica, Universidad Politécnica de Cartagena, Cartagena; j.calatrava@upct.es
}

Resumen: La elevada rentabilidad del regadío en el sudeste de España, unida a unas condiciones climáticas semiáridas, hace que la demanda de agua para riego supere con mucho a la disponibilidad de recursos hídricos, generando una situación de escasez crónica. En este contexto, el reciente desarrollo de la disponibilidad de agua marina desalinizada (AMD) tiene indudables ventajas, al suponer un incremento de la disponibilidad de agua para riego, una mejora de su calidad y una mayor garantía del suministro. Por el contrario, el AMD tiene como mayores inconvenientes su elevado coste y unos mayores costes de fertilización, lo que puede, en principio, reducir la rentabilidad de las explotaciones. En ese sentido, el objetivo de este estudio es analizar la rentabilidad del riego con AMD en el cultivo de tomate en invernadero, uno de los principales cultivos hortícolas del sudeste español, tanto sobre enarenado como en sistemas hidropónicos con reutilización de drenajes.

La información sobre la respuesta agronómica de ambos sistemas de cultivo a los diferentes tipos de agua de riego utilizados (tres mezclas diferentes de AMD con diferentes conductividades eléctricas) procede de un ensayo experimental con tomate bajo plástico realizado dentro de las actividades del proyecto LIFE+ DESEACROP. Para analizar el impacto de los diferentes tratamientos de riego sobre la rentabilidad del cultivo, se ha realizado una caracterización técnico-económica del proceso estándar de producción de tomate para los dos sistemas de producción considerados a partir de estudios previos y de la consulta a técnicos, caracterización en la que se ha integrado la información técnica proveniente del ensayo experimental para calcular diferentes indicadores y ratios económicos.

Los resultados obtenidos muestran cómo el uso exclusivo de AMD para la producción de tomate supone un incremento del $5 \%$ de los costes de fertilización en hidropónico y del 14\% en enarenado y un incremento del $49 \%$ en el coste del agua de riego en cultivo hidropónico y del $54 \%$ en enarenado. Esto se traduce en un incremento de los costes de producción del $6 \%$ en hidropónico y del $4 \%$ en enarenado, incrementos que se ven reducidos de manera significativa cuando se utiliza una mezcla de AMD con aguas subterráneas. Pese a ello, la menor conductividad eléctrica del AMD mejora notablemente los rendimientos del cultivo generando un incremento de su rentabilidad en ambos sistemas de cultivo.

Palabras clave: desalinización; cultivos protegidos; hidropónico; calidad del agua de riego; costes de producción; evaluación económica. 


\title{
Profitability of using desalinated seawater in greenhouse to- mato production in Almeria
}

\author{
Martínez-Granados, D. ${ }^{1}$, Marín-Membrive, P. ${ }^{2}$, Calatrava, J. ${ }^{3}$
}

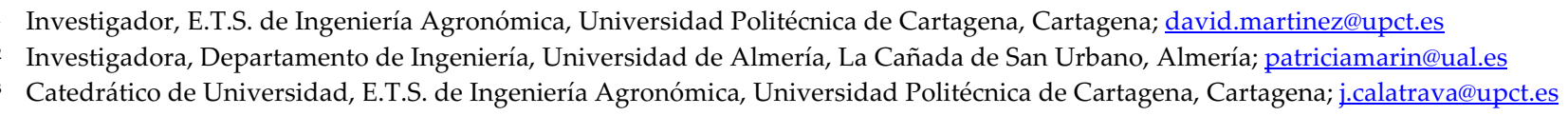

\begin{abstract}
The high profitability of irrigation in south-eastern Spain, coupled with semi-arid climatic conditions, leads to the demand for irrigation water far exceeding the availability of water resources, generating a situation of chronic water shortage. In such context, the recent development of the availability of desalinated seawater (DSW) has undoubted advantages, since it increases the availability of water for irrigation, improves its quality and guarantees a better supply. On the other hand, the main disadvantages of DSW are its high cost and higher fertilization costs, what could, in principle, reduce farm profitability. In this sense, the objective of this study is to analyse the profitability of irrigation with DSW in greenhouse tomato cultivation, one of the main horticultural crops in south-eastern Spain, both on traditional sanded soils and in hydroponic systems with reuse of drainage.
\end{abstract}

The information on the agronomic response of both cropping systems to the different types of irrigation water used (three different DSW mixtures with different electrical conductivities) comes from an experimental trial with tomato under plastic carried out within the activities of the LIFE+ DESEACROP project. To analyse the impact of the different irrigation treatments on crop profitability, a technicaleconomic characterization of the standard tomato production process for the two production systems considered has been carried out based on previous studies and the consultation with technicians, characterization in which the technical information from the experimental trial has been integrated to calculate different indicators and economic ratios.

The results obtained show that the exclusive use of DSW for tomato production increases fertilization costs by $5 \%$ in hydroponics and $14 \%$ in soil production and increases the cost of irrigation water by $49 \%$ in hydroponics and $54 \%$ in soil cultivation. This translates into an increase in production costs of $6 \%$ in hydroponics and $4 \%$ in soil cultivation, increases that are significantly reduced when using a mixture of DSW with groundwater. Despite this cost increase, the lower electrical conductivity of DSW significantly improves crop yields and thus increases profitability for both cropping systems.

Keywords: desalination; protected cultivation; hydroponic; irrigation water quality; production costs; economic assessment. 


\section{Congreso Nacional de Riegos CARTAGENA 2021}

\section{Introducción}

El sudeste de España es una de las regiones con mayor escasez de agua de toda Europa. La elevada rentabilidad de la actividad agrícola, unida a unas condiciones climáticas semiáridas, hace que la demanda de agua para riego supere con mucho a la disponibilidad de recursos hídricos [1], generando una situación de escasez crónica que repercute principalmente sobre la agricultura.

Las respuestas ante esta situación de escasez hidrológica han sido de diversa naturaleza. En primer lugar, la escasez de agua ha incentivado la adopción generalizada de modernos sistemas de riego presurizado, habiéndose producido en paralelo la modernización de gran parte de las infraestructuras de distribución de agua, maximizando su eficiencia a niveles difícilmente mejorables desde un punto de vista técnico [2] y generando importantes beneficios para la agricultura de regadío [3].

La segunda de las respuestas al problema de la escasez de agua en el Sudeste Español ha sido la transferencia de recursos desde otras zonas, a través de los trasvases Negratín-Almanzora y Tajo-Segura, que aportan importantes volúmenes al abastecimiento y al regadío del sudeste de España. Sin embargo, no existen expectativas razonables al respecto de la construcción de nuevos trasvases. Además, las previsiones futuras de disminución de las aportaciones hídricas plantean escenarios claros de reducción de las aportaciones de estos trasvases [4].

En tercer lugar, se ha producido un importante desarrollo de recursos hídricos no convencionales, incluyendo un elevado nivel de reutilización de las aguas residuales urbanas e industriales para aprovechamientos de riego, tanto en la cuenca del Segura como en la provincia de Almería [5, 6], así como el reciente y creciente recurso a la desalinización de agua marina $[7,8]$.

Pese a todas estas actuaciones, la escasez de recursos hídricos continúa siendo una realidad en el sudeste español. Además, las previsiones son que esta escasez se intensifique a medio plazo como consecuencia del cambio climático, con escenarios que prevén una reducción de las aportaciones hídricas medias en régimen natural de entre el 5 y el 11\% [5, 9], lo que supone una amenaza para la continuidad de la agricultura de regadío en muchas zonas. Las opciones de actuación a nivel de explotación pasan por el uso de estrategias de riego más sostenibles basadas en técnicas de riego deficitario controlado, los sistemas de telecontrol para optimizar la gestión del riego, y el recurso a nuevos cultivos o variedades menos demandantes de agua [10,11].

A nivel institucional, el escaso margen existente para mejorar la distribución y aplicación de agua o para incrementar los recursos procedentes de la reutilización (limitados por el consumo urbano e industrial), reduce las alternativas que permitan incrementar la resiliencia de la agricultura de regadío frente al progresivo agotamiento de los sistemas hidrológicos y afrontar la escasez, actual y futura, de agua en el sudeste español. Frente a otras opciones de gestión de la demanda de agua mediante mecanismos económicos, la principal apuesta de la planificación hidrológica nacional para el sudeste ha sido el desarrollo de la disponibilidad de agua de mar desalinizada. Efectivamente, a través del Programa AGUA, España ha hecho en los últimos tres lustros fuertes inversiones para la construcción de plantas desalinizadoras, con el fin de cubrir el déficit hídrico estructural, atendiendo la demanda del regadío y garantizando el suministro urbano.

En la actualidad, en todo el sudeste español, se están suministrando alrededor de $168 \mathrm{hm} /$ año de AMD para riego desde las siete Instalaciones Desalinizadoras de Agua de Mar (IDAM) de las provincias de Alicante, Almería y Murcia que suministran para uso agrícola (Torrevieja, Escombreras, Valdelentisco, Águilas-Guadalentín, Cuevas de Almanzora, Carboneras y Campo de Dalías). En el caso de la cuenca del Segura, durante los primeros años de operación de las IDAM, la demanda de AMD para uso agrícola fue reducida, manteniéndose entre 20 y $25 \mathrm{hm}^{3} /$ año hasta 2012. A partir de 2013, el uso de AMD desalinizada empieza a crecer, alcanzando los 139 hm³/año en 2016, con previsiones de alcanzar 


\section{Congreso Nacional de Riegos CARTAGENA 2021}

los $175 \mathrm{hm} 3 /$ año en pocos años. Este auge del uso del AMD para el riego agrícola en el sudeste español se explica por varias circunstancias favorables: el gran número de IDAMs financiadas por el Programa AGUA, que además utilizan tecnologías actuales y bastante eficientes, lo que reduce el coste de producción del AMD; la creciente necesidad de aportar nuevos recursos hídricos que ayuden a paliar el déficit estructural de agua; y la elevada rentabilidad de la agricultura de regadío en determinadas zonas.

Este hecho tiene indudables ventajas para el regadío del sudeste español, ya que supone una mayor cantidad de agua disponible para la producción agrícola, una mejora de la calidad del agua de riego en algunas zonas y un incremento de la garantía del suministro. Por el contrario, el agua marina desalinizada tiene como mayor inconveniente el elevado coste de su suministro, lo que reduce la rentabilidad de la actividad agraria. Asimismo, el AMD presenta también problemas que derivan, principalmente, de su baja concentración en nutrientes como calcio, magnesio y sulfato, que son esenciales para el desarrollo de los cultivos, y cuya presencia en las aguas continentales hace innecesario su aporte mediante fertilización [12-14]. Estas carencias obligan a realizar aportes de estos nutrientes, lo que incrementa los costes de la fertilización y tiene un impacto claro sobre la rentabilidad de las explotaciones [15].

En ese sentido, el objetivo de este estudio es analizar la rentabilidad y productividad de los principales factores de producción del riego con agua marina desalinizada en el cultivo de tomate en invernadero, uno de los principales cultivos hortícolas del sudeste español, tanto sobre enarenado como en sistemas hidropónicos con reutilización de drenajes prestando especial atención al impacto del coste del AMD y de la fertilización.

\section{Materiales y métodos}

La información sobre la respuesta del cultivo de tomate al riego con agua marina desalinizada, tanto sobre enarenado como en sistemas hidropónico con reutilización de drenajes, proviene de un ensayo experimental llevado a cabo en un invernadero tipo Almería, con ventilación cenital y lateral natural automatizada y sin calefacción, localizado en la Fundación Finca Experimental UALANECOOP, Retamar (Almería). Dicho ensayo se enmarca dentro de las actividades del proyecto LIFE+ DESEACROP (Desalinated Seawater for Alternative and Sustainable Soilless Crop Production) que se centra en la demostración del uso de técnicas de manejo eficiente y sostenible del agua marina desalinizada en sistemas de producción de tomate sin suelo de la horticultura almeriense. Con dicho objetivo en mente, las actividades experimentales del mencionado proyecto incluyeron diferentes tratamientos de riego utilizando diferentes tipos de agua en sistemas hortícolas protegidos, desarrollándose el cultivo tanto sobre suelo como en hidropónico. Los ensayos experimentales se han realizado durante dos ciclos otoño-invierno de tomate (Solanum lycopersicum L. cv. Ramyle) y dos ciclos primavera-verano de tomate (Solanum lycopersicum L. cv. Ramyle).

Para analizar el efecto del sistema de cultivo se han considerado [16]: 1) un sistema de cultivo hidropónico sobre sustrato de fibra de coco con reutilización de los drenajes; y 2) un sistema de enarenado tradicional de la zona sin reutilización de drenajes. Para analizar el efecto del riego con agua marina desalinizada, frente a las fuentes de suministro habitual en la zona, se han considerado tres tipos tratamientos de riego [17]: 1) agua marina desalinizada proveniente de la IDAM de Carboneras (tratamiento T1); 2) una mezcla de agua subterránea y AMD con una conductividad eléctrica de 1,5 dS/m (tratamiento T2); y 3) una mezcla de agua subterránea y AMD con una conductividad eléctrica de $3 \mathrm{dS} / \mathrm{m}$ (tratamiento T3).

Para analizar el impacto de los diferentes tratamientos de riego considerados sobre la rentabilidad del cultivo analizado, se ha partido de una caracterización técnico-económica del proceso estándar de 


\section{Congreso Nacional de Riegos CARTAGENA 2021}

producción de tomate para los dos sistemas de producción considerados. Dicha caracterización se ha realizado a partir información secundaria proveniente de estudios previos [18-20] y de la consulta a técnicos, y se ha contrastado con el proceso de producción llevado a cabo en el ensayo experimental para asegurar su compatibilidad. La caracterización técnico-económica de los procesos de producción en términos de cantidad y calidad de la producción, uso de insumos (incluyendo mano de obra), costes de producción, dinámica del proceso de producción, etc. requiere de la obtención de una amplia información de las diferentes labores u operaciones que se llevan a cabo durante el ciclo de cultivo. Para ello, las operaciones de cultivo se han organizado por meses, empezando inmediatamente después de la recolección y finalizando en la recolección de la siguiente campaña, y se han desagregado por tipos de operaciones (labores de preparación del terreno y del cultivo, labores culturales, aplicación del riego y de diversos tratamientos y tareas de recolección). Asimismo, se ha caracterizado la estructura productiva de una explotación estándar de la zona de estudio en términos de infraestructuras, sistemas de cultivo y riego, etc.

La caracterización técnico-económica del proceso productivo realizada para cada uno de los sistemas de cultivo considerados se ha utilizado para analizar el impacto sobre su rentabilidad del uso de cada tipo de recursos hídricos (tratamientos) considerados en el estudio. Para ello, se ha recogido información del ensayo experimental que se ha integrado en dicha caracterización técnico-económica y se han calculado una serie de indicadores y ratios económicos. Se presentan en este trabajo los resultados correspondientes a la media para los cuatro ciclos de cultivo de los diferentes tratamientos ensayados.

\section{Resultados y discusión}

\subsection{Rendimientos}

La Tabla 1 resume los valores medios de los indicadores calculados. Para interpretarlos correctamente, es importante considerar que los rendimientos del cultivo obtenidos en las actividades experimentales del proyecto están por debajo de los rendimientos normales del tomate en Almería. Esto se debe a la necesidad de incorporar más de dos ciclos productivos en la vida del proyecto, lo que llevó a la decisión de plantar cuatro ciclos productivos cortos en lugar de dos largos. Además, la necesidad de encajar dos ciclos productivos de tomate en un año llevó a terminar la recolección del cultivo antes de lo que es habitual en las explotaciones comerciales, para dar espacio y tiempo a la preparación del siguiente ciclo experimental. Esto ha dado lugar a un rendimiento medio anual del cultivo $\left(11 \mathrm{~kg} / \mathrm{m}^{2}\right)$ inferior al habitual en la zona que es de $19 \mathrm{~kg} / \mathrm{m}^{2}$ al año [21].

Los rendimientos medios de tomate en la Tabla 1 muestran un impacto positivo tanto de la calidad del agua como del uso de sistemas productivos sin suelo. El aumento del rendimiento medio del cultivo al pasar de T3 a T2 es significativo, mientras que la diferencia entre T2 y T1 es menor. El aumento del rendimiento de los cultivos al regar con agua menos salina es significativamente mayor para la producción sin suelo con recirculación de los flujos de drenaje $(\mathrm{H})$ que para el cultivo en enarenado. La mayor diferencia en el rendimiento de los cultivos entre T2 y T3 para el cultivo sin suelo $(\mathrm{H})$ con respecto al cultivo en suelo $(S)$ puede explicarse por la naturaleza más compleja de la tecnología de cultivo sin suelo. Los sistemas tradicionales de producción en suelo de la zona se desarrollaron para adaptarse a suelos pobres y recursos hídricos de mala calidad y, por tanto, los rendimientos se ven menos afectados por la salinidad del agua, que reduce significativamente los rendimientos en el cultivo sin suelo. 


\section{Congreso Nacional de Riegos CARTAGENA 2021}

\subsection{Costes de producción}

Los costes de producción, tanto directos como indirectos, son mayores para el cultivo sin suelo $(\mathrm{H})$ que para el cultivo tradicional en suelo (S), como se muestra en la Tabla 1. Por su parte, un mayor uso de AMD incrementa solamente los costes directos (T1>T2>T3). Las diferencias en los costes directos se explican por el mayor coste del AMD, el mayor consumo de agua, energía y fertilizantes de la producción sin suelo $(\mathrm{H})$ y el coste de las labores de recolección que depende del rendimiento del cultivo. El sistema de producción sin suelo tiene la ventaja de evitar la percolación de nutrientes al suelo, ya que el drenaje se recircula, pero al mismo tiempo consume más agua, fertilizantes y energía. Por otro lado, el rendimiento de los cultivos también aumenta con la producción sin suelo. Las diferencias en los costes indirectos se explican por el coste de amortización del sustrato y del sistema de recirculación de los drenajes.

Debido a lo comentado anteriormente, los costes unitarios de producción por kilogramo son menores para la producción sin suelo $(\mathrm{H})$ en T1 y T2, mientras que son mayores para el agua más salina (T3) debido a los menores rendimientos obtenidos. Un patrón similar se observa cuando se examina el coste unitario de la mano de obra. Por otro lado, los costes unitarios de agua, energía y fertilización son mayores para la producción sin suelo $(\mathrm{H})$ que para la producción en suelo (S). Esto se debe a que el consumo de agua, energía y fertilizantes es significativamente mayor en la producción sin suelo, a pesar de los mayores rendimientos que proporciona este sistema productivo. Sin embargo, el coste de estos insumos es relativamente pequeño en comparación con otros insumos como el capital y la mano de obra y se ve compensado, en general, por el mayor rendimiento de los cultivos que proporcionan los sistemas sin suelo.

\subsection{Productividades de los factores de producción}

La Tabla 1 muestra en primer lugar que tanto la productividad de la tierra como la del trabajo son mayores en la producción sin suelo $(\mathrm{H})$ para las calidades de agua T1 y T2. De nuevo, los resultados para T3 en la producción sin suelo son peores que en la producción con suelo tradicional, debido a las diferencias antes mencionadas en el rendimiento de los cultivos. Para cada sistema productivo, tanto la productividad de la tierra como la del trabajo aumentan con la calidad del agua (T1 $>$ T2 $>$ T3).

Por el contrario las productividades tanto de agua como de energía son mayores para la producción en suelo tradicional que para la produccón sin suelo, lo que se debe a las mayores necesidades de agua y energía de la producción sin suelo. El incremento de los rendimientos en hidropónico con respecto al cultivo en enarenado es menor que los incrementos en el consumo de agua y energia asociados. Finalmente, se observa cómo, para un mismo sistema productivo, las productividades aumentan con la calidad del agua (T1>T2>T3).

\subsection{Indicadores de rentabilidad}

En cuanto a las diferentes indicadores de rentabilidad calculados, su interpretación debe considerar que algunas de ellas toman valores negativos debido a los rendimientos inferiores a los habituales, ya comentados, obtenidos en los experimentos del proyecto. Para todas las medidas de rentabilidad consideradas (tierra, agua y energía), la rentabilidad por unidad aumenta con la calidad del agua (T1 $>\mathrm{T} 2>\mathrm{T} 3$ ). Todas las medidas de rentabilidad son también mayores para la producción sin suelo $(\mathrm{H})$ para T1 y T2, pero lo contrario ocurre para T3, debido al impacto de la baja calidad del agua en los rendimientos de los cultivos para la producción sin suelo (Tabla 1). 


\section{Congreso Nacional de Riegos CARTAGENA 2021}

Tabla 1. Indicadores económicos calculados para las diferentes estrategias analizadas

\begin{tabular}{|c|c|c|c|c|c|c|c|}
\hline & \multirow{2}{*}{ INDICADOR } & \multicolumn{6}{|c|}{ ESTRATEGIAS } \\
\hline & & H-T1 & H-T2 & H-T3 & S-T1 & S-T2 & S-T3 \\
\hline $\begin{array}{l}\text { Rendimiento del } \\
\text { cultivo }\end{array}$ & $\begin{array}{l}\text { Rendimiento medio del cultivo } \\
\qquad(\mathrm{kg} / \mathrm{ha})\end{array}$ & 66438 & 64375 & 44675 & 55378 & 54311 & 47353 \\
\hline \multirow{8}{*}{ Costes } & Costes directos $(€ /$ ha $)$ & 39837 & 39712 & 37458 & 36577 & 36494 & 35280 \\
\hline & Costes indirectos $(€ /$ ha $)$ & 11897 & 11897 & 11897 & 10158 & 10158 & 10158 \\
\hline & Costes de producción totales (€/ha) & 51734 & 51609 & 49355 & 46735 & 46652 & 45438 \\
\hline & $\begin{array}{c}\text { Costes unitarios de producción } \\
(€ / \mathrm{kg})\end{array}$ & 0,7787 & 0,8017 & 1,1048 & 0,8439 & 0,8590 & 0,9596 \\
\hline & Costes unitarios del agua $(€ / \mathrm{kg})$ & 0,0169 & 0,0166 & 0,0169 & 0,0148 & 0,0138 & 0,0112 \\
\hline & Costes energéticos unitarios $(€ / \mathrm{kg})$ & 0,0173 & 0,0185 & 0,0239 & 0,0009 & 0,0009 & 0,0009 \\
\hline & $\begin{array}{l}\text { Costes unitarios de fertilización } \\
(€ / \mathrm{kg})\end{array}$ & 0,0415 & 0,0435 & 0,0591 & 0,0328 & 0,0348 & 0,0336 \\
\hline & $\begin{array}{l}\text { Coste unitario de la mano de obra } \\
\qquad(€ / \mathrm{kg})\end{array}$ & 0,3509 & 0,3597 & 0,4813 & 0,4046 & 0,4109 & 0,4566 \\
\hline \multirow{4}{*}{ Productividades } & Productividad de la tierra (€/ha) & 55458 & 53736 & 37292 & 46226 & 45336 & 39528 \\
\hline & Productividad del agua $\left(€ / \mathrm{m}^{3}\right)$ & 23,60 & 22,01 & 17,06 & 27,03 & 26,58 & 25,82 \\
\hline & $\begin{array}{l}\text { Productividad de la mano de obra } \\
(€ / \text { día })\end{array}$ & 142,7 & 139,2 & 104,1 & 123,8 & 121,9 & 109,7 \\
\hline & Productividad de la energía $(€ /$ kWh $)$ & 5,34 & 4,98 & 3,86 & 108,12 & 106,31 & 103,28 \\
\hline \multirow{6}{*}{ Rentabilidades } & Margen bruto por hectárea $(€ /$ ha $)$ & 15621 & 14024 & -166 & 9649 & 8842 & 4247 \\
\hline & Margen neto por hectárea $(€ /$ ha $)$ & 4451 & 2854 & -11336 & 218 & -589 & -5184 \\
\hline & $\begin{array}{l}\text { Margen bruto por unidad de agua } \\
\qquad\left(€ / \mathrm{m}^{3}\right)\end{array}$ & 6,65 & 5,74 & $-0,08$ & 5,64 & 5,18 & 2,77 \\
\hline & $\begin{array}{l}\text { Margen neto por unidad de agua } \\
\left(€ / \mathrm{m}^{3}\right)\end{array}$ & 1,89 & 1,17 & $-5,19$ & 0,13 & $-0,35$ & $-3,39$ \\
\hline & $\begin{array}{c}\text { Margen bruto por uso de energía } \\
(€ / \mathrm{kWh})\end{array}$ & 1,50 & 1,30 & $-0,02$ & 22,57 & 20,73 & 11,10 \\
\hline & $\begin{array}{l}\text { Margen neto por uso de energía } \\
(€ / \mathrm{kWh})\end{array}$ & 0,43 & 0,26 & $-1,17$ & 0,51 & $-1,38$ & $-13,54$ \\
\hline \multirow{3}{*}{$\begin{array}{l}\text { Uso de mano de } \\
\text { obra }\end{array}$} & $\begin{array}{l}\text { Uso de mano de obra por hectárea } \\
\text { (días/ha) }\end{array}$ & 389 & 386 & 358 & 373 & 372 & 360 \\
\hline & $\begin{array}{l}\text { Uso de mano de obra por unidad de } \\
\left.\text { agua (días } / \mathrm{m}^{3}\right)\end{array}$ & 0,1653 & 0,1581 & 0,1640 & 0,2183 & 0,2180 & 0,2354 \\
\hline & $\begin{array}{l}\text { Uso de mano de obra por kWh } \\
\text { (días } / \mathrm{kWh} \text { ) }\end{array}$ & 0,0374 & 0,0358 & 0,0371 & 0,8733 & 0,8721 & 0,9415 \\
\hline
\end{tabular}

\subsection{Impacto social}

Pasando a los indicadores sociales que contemplan la demanda de mano de obra por unidad de los diferentes factores de producción, la Tabla 1 muestra que la mejora de la calidad del agua mediante el uso de AMD y el uso de sistemas productivos sin suelo con recirculación de drenaje da lugar a un 


\section{Congreso Nacional de Riegos CARTAGENA 2021}

ligero aumento del uso de mano de obra por hectárea, pero con diferencias muy pequeñas para las diferentes fuentes de agua (T1, T2 y T3). Por el contrario, el uso más intensivo del agua y de la energía en los sistemas de cultivo sin suelo reduce el uso de mano de obra por $\mathrm{m}^{3} \mathrm{y}$ por $\mathrm{kWh}$ con respecto al cultivo de suelo convencional.

\section{Conclusiones}

En este trabajo se presentan resultados de la evaluación del impacto económico del riego con agua marina desalinizada en el cultivo de tomate en invernadero, tanto sobre enarenado como en sistemas hidropónicos con reutilización de drenajes, de la provincia de Almería. Los resultados presentados se centran en el análisis de las diferentes estrategias analizadas en las actividades experimentales del proyecto DESEACROP, en las que se ha comparado el uso de diferentes fuentes de agua en sistemas protegidos de producción de tomate, tanto tradicionales como sin suelo.

Los resultados obtenidos ponen de manifiesto, en primer lugar, como el uso de AMD incrementa los costes de producción pero también los rendimiento de los cultivos, al reducirse la salinidad del agua, dando lugar a una mayor rentabilidad de los mismos. El uso exclusivo de AMD para la producción de tomate supone un incremento del $5 \%$ de los costes de fertilización en hidropónico y del $14 \%$ en enarenado y un incremento del $49 \%$ en el coste del agua de riego en cultivo hidropónico y del $54 \%$ en enarenado. Esto se traduce en un incremento de los costes de producción del $6 \%$ en hidropónico y del $4 \%$ en enarenado, incrementos que se ven reducidos de manera significativa cuando se utiliza una mezcla de AMD con aguas subterráneas. Pese a ello, la menor conductividad eléctrica del AMD mejora los rendimientos del cultivo generando un incremento de su rentabilidad. Asimismo, todas las productividades calculadas aumentan con la mejora de la calidad del agua gracias al mayor uso de AMD.

En cuanto al sistema de cultivo, el cultivo sin suelo es más intensivo en cuanto al uso de insumos, especialmente agua, energía y fertilizantes, lo que se traduce en mayores costes de producción que se compensan con un mayor rendimiento de los cultivos y una mayor rentabilidad de los mismos. Sin embargo, el rendimiento y la rentabilidad de los cultivos cuando se utiliza más agua salina son mayores para el sistema de producción tradicional en suelo. La materialización de los beneficios potenciales de la producción sin suelo en términos de rendimiento y rentabilidad de los cultivos requiere, por tanto, la utilización de recursos hídricos de mejor calidad. Asimismo, el uso de sistemas de producción sin suelo aumenta la productividad de la tierra y del trabajo con respecto a los sistemas de suelo tradicionales, pero da lugar a productividades más bajas de los factores agua y energía.

En cuanto a la demanda de mano de obra, tanto el uso de sistemas de producción sin suelo como el riego con AMD aumenta las necesidades de de mano de obra por hectárea, pero reduce el uso de mano de obra por $\mathrm{m}^{3} \mathrm{y}$ por $\mathrm{kWh}$.

En resumen, los resultados de las actividades experimentales del proyecto sugieren que tanto el uso de AMD como los sistemas de producción sin suelo aumentarían la rentabilidad de las explotaciones en la zona de estudio. Sin embargo, la materialización de los beneficios potenciales de la producción sin suelo requiere el uso de recursos hídricos de mejor calidad. En la zona de estudio, donde los recursos hídricos naturales disponibles son altamente salinos, la mejora de la calidad del agua de riego implica el uso de AMD. De lo contrario, el sistema tradicional de producción en suelo con enarenado, mejor adaptado a los suelos pobres y al agua de mala calidad, sería más rentable.

Sin embargo, desde el punto de vista de la sociedad, las ventajas de la producción sin suelo y del AMD son ambiguas. Mientras que el uso de AMD mejora la productividad de los insumos y, por tanto, la eficiencia en el uso de los recursos, el uso de sistemas de producción sin suelo reduce la 


\section{Congreso Nacional de Riegos CARTAGENA 2021}

productividad del agua y la energía, que son recursos escasos. Si nos fijamos en la generación de mano de obra, tanto la producción sin suelo como el AMD generan menos mano de obra por uso de agua y energía. La intensificación de la producción de tomate mediante el uso de tecnologías de producción sin suelo aumenta el uso de recursos productivos muy limitantes (agua y energía) sin que el aumento de la producción y la generación de empleo resultantes lo compensen. Sin embargo, esto podría ser compensado por la reducción de la lixiviación y, por tanto, de la contaminación del suelo y del agua, un efecto que no se evalúa aquí.

Es necesario indicar, finalmente, que los resultados de este trabajo se ven afectados cuantitativamente por las restricciones específicas del entorno experimental que dieron lugar a rendimientos de los cultivos inferiores a la media de la zona, pero siguen siendo válidos cualitativamente en lo que se refiere a la influencia de los factores (calidad del agua y sistema de cultivo) analizados.

\section{Agradecimientos}

Este trabajo ha sido financiado por la Unión Europea a través del Proyecto LIFE+ DESEACROP (LIFE16 ENV/ES/000341).

\section{Referencias}

1. Urquijo, J.; De Stefano, L. Perception of Drought and Local Responses by Farmers: A Perspective from the Jucar River Basin, Spain. Water Resour Manag 2016, 30, 577-591.

2. Soto-García, M.; Martínez-Alvarez, V.; García-Bastida, P.A.; Alcon, F.; Martin-Gorriz, B. Effect of water scarcity and modernisation on the performance of irrigation districts in south-eastern Spain. Agr Water Manage 2013, 124, 11-19.

3. Berbel, J.; Gutiérrez-Martín, C., Eds. Efectos de la modernización de regadíos en España. Serie Economía nº 30; Cajamar Caja Rural: Almería, España, 2017.

4. Senent-Aparicio, J.; López-Ballesteros, A.; Cabezas, F.; Pérez-Sánchez, J.; Molina-Navarro, E. A Modelling Approach to Forecast the Effect of Climate Change on the Tagus-Segura Interbasin Water Transfer. Water Resour Manage 2021, 35, 37913808.

5. CHS. Plan Hidrológico de la Cuenca del Segura 2015-2021; Confederación Hidrográfica de la Cuenca del Segura: Murcia, España, 2015.

6. Valera, D.L.: Belmonte, L.J.; Molina-Aiz, F.D.; López, A. Greenhouse Agriculture in Almería. A Comprehensive Techno-Economic Analysis; Cajamar Caja Rural: Almería, España, 2016.

7. Martínez-Álvarez, V.; González-Ortega, M.J.; Martin-Gorriz B.; Soto-García, M.; Maestre-Valero, J. F. The use of desalinated seawater for crop irrigation in the Segura River Basin (south-eastern Spain). Desalination 2017, 422, 153-164.

8. Melgarejo-Moreno, J.; López-Ortiz, M. I.; Fernández-Aracil, P. Water distribution management in South-East Spain: A guaranteed system in a context of scarce resources. Sci Total Environ 2019, 648, 1384-1393.

9. CEDEX. Evaluación del cambio climático en los recursos hídricos en régimen natural en España; Centro de Estudios y Experimentación de Obras Públicas: Madrid, España, 2011.

10. Fereres, E.; Soriano, M.A. Deficit irrigation for reducing agricultural water use. J Exp Bot 2007, 58(2), 147-159.

11. Ruiz-Sanchez, M. C.; Domingo, R.; Castel, J.R. Review. Deficit irrigation in fruit trees and vines in Spain. Span J Agric Res 2010, 8(S2), 5-20.

12. Yermiyahu, U.; Tal, A.; Ben-Gal, A.; Bar-Tal, A.; Tarchitzky, J.; Lahav, O. Rethinking desalinated water quality and agriculture. Science, 2007, 318, 920-921.

13. Ben-Gal, A.; Yermiyahu, U.; Cohen, S. Fertilization and blending alternatives for irrigation with desalinated water. J Environ Qual 2009, 38, 529-536.

14. Martínez-Alvarez, V.; Martin-Gorriz, B.; Soto-García, M. Seawater desalination for crop irrigation - A Review of current experiences and revealed key issues. Desalination 2016, 381, 58-70.

15. Maestre-Valero, J.F.; Martin-Gorriz, B.; Soto-García, M.; Martinez-Mate, V.; Martínez-Álvarez, V. Producing lettuce in soilbased or in soilless outdoor systems. Which is more economically profitable? Agr Water Manage 2018, 206, 48-55.

16. UAL. Deliverable DB1.1: Report on the configuration of the demonstrative plots; LIFE+ DESEACROP Project, Reference LIFE 16ENV-ES-000341, Universidad de Almería: Almería España, 2018. 


\section{Congreso Nacional de Riegos CARTAGENA 2021}

17. UAL. Deliverable DB2.1: Report on irrigation treatments and procedures to the water quality adjustments; LIFE+ DESEACROP Project, Reference LIFE 16-ENV-ES-000341, Universidad de Almería: Almería, España, 2018.

18. MAGRAMA. Estudios de Costes y Rentas de las Explotaciones Agrarias: Resultados técnico-económicos; Ministerio de Agricultura, Alimentación y Medio Ambiente: Madrid, España, 2015.

19. Junta de Andalucía. Anuario de estadísticas agrarias y pesqueras de Andalucía; Consejería de Agricultura, Ganadería, Pesca y Desarrollo Sostenible, Junta de Andalucía: Sevilla, España, 2018.

20. Calatrava, J.; Martínez-Granados, D. La productividad económica en los sistemas agrarios intensivos del Mediterráneo. In El regadío en el Mediterráneo Español. Serie Monografías nº 38; Garrido, A., Pérez Pastor, A., Eds.; Cajamar Caja Rural: Almería, España, 2019; pp. 97-124.

21. Valera D.; Belmonte L.J.; Molina F.D.; López A. Los invernaderos de Almería: análisis de su tecnología y rentabilidad; Cajamar Caja Rural: Almería, España, 2014. 